\title{
Benzodiazepines, z-Hypnotics, and Risk of Dementia: Special Considerations of Half-Lives and Concomitant Use
}

\author{
Li-Yen Tseng $^{1,2,3} \cdot$ Shih-Tsung Huang ${ }^{4} \cdot$ Li-Ning Peng $^{1,2,3} \cdot$ Liang-Kung Chen $^{1,2,3} \cdot$ Fei-Yuan Hsiao $^{4,5,6} \mathbb{C}$
}

Published online: 4 December 2019

(C) The American Society for Experimental NeuroTherapeutics, Inc. 2019

\begin{abstract}
The utilization of benzodiazepines (BZDs) and z-hypnotics has substantially increased with the aging of the population, but the risk of BZDs and z-hypnotics in the development of dementia remains a strong concern. This cohort study aimed to evaluate the risk of BZDs and z-hypnotics for subsequent dementia development with a special consideration of their half-lives and the concomitant use of these medications. People aged 65 years and older who were newly prescribed oral BZDs or Z-hypnotics between 2003 and 2012 were identified from Taiwan's National Health Insurance Research Database. All BZDs were categorized as long-acting drugs $(\geq 20 \mathrm{~h})$ or short-acting drugs $(<20 \mathrm{~h})$ for further comparisons, and data were collected on a quarterly basis, starting on the first date of drug prescription and ending on the date of death, occurrence of dementia, or end of the follow-up period (December 31, 2012), whichever came first. All dementia events except vascular dementia occurring during the follow-up period were identified. Among 260,502 eligible subjects, short-acting BZDs and z-hypnotics users were at greater risk of dementia than long-acting users [adjusted odds ratio (95\% confidence interval) in short-acting BZD users, 1.98 (1.89-2.07); z-hypnotic users, 1.79 (1.68-1.91); and long-acting BZD users, 1.47 (1.37-1.58)]. In addition, subjects concomitantly using 2 or more BZDs or z-hypnotics had a higher risk of dementia than those who used 1 of these drugs $(4.79$ (3.95-5.81)). The use of BZDs and z-hypnotics was strongly associated with the risk of dementia development, especially the short-acting BZDs, zhypnotics, and concomitant use of multiple agents. These findings deserve further interventional studies for clarification.
\end{abstract}

Key Words Benzodiazepines $\cdot$ z-hypnotics $\cdot$ dementia $\cdot$ elderly $\cdot$ half-life

Li-Yen Tseng and Shih-Tsung Huang contributed equally to this work.

Electronic supplementary material The online version of this article (https://doi.org/10.1007/s13311-019-00801-9) contains supplementary material, which is available to authorized users.

Fei-Yuan Hsiao

fyshsiao@ntu.edu.tw

1 Department of Geriatrics, School of Medicine, National Yang-Ming University, Taipei, Taiwan

2 Aging and Health Research Center, National Yang-Ming University, Taipei, Taiwan

3 Center for Geriatrics and Gerontology, Taipei Veterans General Hospital, Taipei, Taiwan

4 Graduate Institute of Clinical Pharmacy, College of Medicine, National Taiwan University, 33, Linsen S. Rd, Taipei 10050, Taiwan

5 School of Pharmacy, College of Medicine, National Taiwan University, Taipei, Taiwan

6 Department of Pharmacy, National Taiwan University Hospital, Taipei, Taiwan

\section{Introduction}

Benzodiazepines (BZDs) and z-hypnotics (such as zolpidem) are widely prescribed to older adults for various purposes, either alone or in combination with other disease-related treatments [1-4]. Despite the risk of BZDor z-hypnotic-related falls, fractures, traffic accidents, and cognitive dysfunction [5-7], considerable research and public attention have focused on the association between BZDs or z-hypnotics and the risk of dementia [5, 8-14]. However, this association remains less well understood, and symptoms that BZDs or z-hypnotics are indicated for may be the prodromal symptoms of dementia. The use of BZDs and zhypnotics may result in acute cognitive decline [15], but evidence regarding their long-term use and cognitive decline varies greatly [16-21]. Although some studies have suggested that BZDs and z-hypnotics may not have long-term effects [16, 17], others have reported a potential association between the use of BZDs and z-hypnotics and the subsequent development of dementia or Alzheimer's disease [22, 23]. 
These discrepancies in previous studies may have resulted from different methodological limitations. First, available reports often group all BZDs together without consideration of their pharmacological properties, such as their half-lives, i.e., short- or long-acting BZDs [11, 12]. Second, many existing studies have often investigated the association between individual BZDs or z-hypnotics and the risk of dementia [8-12]. However, older adults often switch their medications between the 2 classes of drugs, as well as between short- and long-acting BZDs. These utilization patterns made it difficult to capture the actual effects of long-term use of BZDs and/or z-hypnotics and risk of dementia. Novel techniques to capture the real-world utilization patterns of BZDs or z-hypnotics are, thus, critical to fill the knowledge gap regarding the associations between BZDs or z-hypnotics and risk of dementia.

To overcome these limitations of previous studies, we conducted a longitudinal cohort study to investigate the association between BZDs or z-hypnotics and the risk of dementia by adopting time-varying (quarterly) measures of exposure to BZDs or z-hypnotics. Furthermore, we also aimed to test the hypothesis that BZDs with different half-lives may have a differential impact on dementia risk.

\section{Methods}

\section{Data Source}

A population-based retrospective longitudinal cohort study was conducted using administrative health data from Taiwan's National Health Insurance Research Database (NHIRD). The NHIRD is a nationwide claims-based database comprising anonymous eligibility and enrollment information, as well as claims for outpatient visits, admissions, procedures, and prescription medications of more than $99 \%$ of the entire population (23 million) in Taiwan [24]. We used a subset of the NHIRD, which contains claims data for $20 \%$ of randomly selected beneficiaries who were aged 65 years old and older from 2004 to 2008 to create a 10-year (2003-2012) panel of claims for analysis.

\section{Ethical Statement}

The identification numbers for all of the entries in the NHIRD were encrypted to protect the privacy of the individual patients. The study protocol was approved by the Institution Review Board of the National Taiwan University Hospital (National Taiwan University Hospital Research Ethics Committee No. 201403069W).

\section{Study Population}

From the NHIRD, we identified elderly individuals aged 65 years and older who initiated the use of oral BZDs or zhypnotics in an outpatient setting between 2003 and 2012 as our study cohort. The index date was defined as the date of first prescription of oral BZDs or z-hypnotics. The last year before the index date served as the baseline informationtaking period. The follow-up period for each study subject started on the index date and ended on the date of death, the date of a dementia event, or December 31, 2012, whichever came first. Those who had ever received oral BZDs or zhypnotics and were diagnosed with dementia in the outpatient or inpatient setting within 1 year prior to the index date were excluded. To prevent lag time bias, we also excluded patients who had any diagnosis of dementia in the outpatient or inpatient setting in the first year of the follow-up period. In addition, to explore the risk of drug-related dementia events, we excluded individuals who had vascular dementia (with an International Classification of Disease, 9th Edition, diagnosis code (ICD-9-CM codes) 290.4) or those who were diagnosed with dementia within 1 year after stroke occurring during the follow-up period.

\section{Drug Exposure}

We assumed that the use of BZDs and z-hypnotics was a dynamic process since older persons may discontinue, switch, or reinitiate the use of these drugs very frequently; therefore, we measured BZD and z-hypnotic use quarterly to identify the actual utilization of BZDs and z-hypnotics during the followup period. In addition, as we intended to test the hypothesis that half-lives of BZDs were associated with different risk levels of dementia, we distinguished between the use of long-acting BZDs (half-life $\geq 20 \mathrm{~h}$ ) and short-acting BZDs (half-life $<20 \mathrm{~h}$ ) according to their half-lives $[25,26]$ (Table 1), and this cutoff time was based on previous studies $[9,10]$.

Exposure to BZDs and z-hypnotics was defined as receiving at least 28 days of the same half-life class of BZDs or zhypnotics in a quarter and recorded as a time-varying binary variable. Moreover, considering that some subjects may use more than 2 classes of BZDs or z-hypnotics in the same quarter, we further categorized the utilization pattern of BZDs and Z-hypnotics as 1) nonuse, 2) only long-acting BZDs, 3) only short-acting BZDs, 4) only z-hypnotics, 5) long-acting BZDs + short-acting BZDs (2 combined), 6) long-acting BZDs + zhypnotics (2 combined), 7) short-acting BZDs + z-hypnotics ( 2 combined), and 8 ) long-acting BZDs + short-acting BZDs + z-hypnotics (3 combined). For sensitivity analysis, we changed the definition of drug exposure as the use of the same drug class for at least 1 day, 7 days, or 14 days in each quarter. 
Table 1 Half-life and Anatomical Therapeutic Chemical (ATC) code for benzodiazepine and z-drug

\begin{tabular}{|c|c|c|}
\hline Type & Drug name (half-life, in h) & ATC code \\
\hline \multirow{12}{*}{$\begin{array}{l}\text { Long-acting } \\
\quad \text { (half-life } \geq 20 \mathrm{~h})\end{array}$} & Bromazepam (20.6) & N05BA08 \\
\hline & Chlordiazepoxide (24-48) & N05BA02 \\
\hline & Clobazam (36-42) & N05BA09 \\
\hline & Clonazepam $(30-40)$ & N03AE01 \\
\hline & Diazepam (48) & N05BA01 \\
\hline & Nitrazepam (24-29) & N05CD02 \\
\hline & Prazepam (78) & N05BA11 \\
\hline & Medazepam (36-200) & N05BA03 \\
\hline & Nordazepam (36-200) & N05BA16 \\
\hline & $\begin{array}{l}\text { Flunitrazepam (18-26 but } \\
36-200 \text { for active metabolite) }\end{array}$ & $\mathrm{N} 05 \mathrm{CD} 03$ \\
\hline & Oxazolam (61.2) & N05BA91 \\
\hline & Brotizolam (4-8) & N05CD09 \\
\hline \multirow{10}{*}{$\begin{array}{l}\text { Short-acting } \\
\quad \text { (half-life }<20 \text { h) }\end{array}$} & Alprazolam (10.7-15.8) & N05BA12 \\
\hline & Clorazepate (2.3) & N05BA05 \\
\hline & Estazolam (10-24) & N05CD04 \\
\hline & Flurazepam (2.3) & N05CD01 \\
\hline & Lorazepam (12-14) & N05BA06 \\
\hline & Midazolam (1.8-6.4) & N05CD08 \\
\hline & Oxazepam (5.6-10.9) & N05BA04 \\
\hline & Temazepam (3.5-18.4) & N05CD07 \\
\hline & Triazolam $(1.5-5.5)$ & N05CD05 \\
\hline & Lormetazepam (10-12) & N05CD06 \\
\hline \multirow[t]{4}{*}{ z-drug } & Zolpidem (2.5-2.6) & N05CF02 \\
\hline & Zaleplon (1) & N05CF03 \\
\hline & Zopiclone (3.5-6.5) & N05CF01 \\
\hline & Eszopiclone (6-9) & N05CF04 \\
\hline
\end{tabular}

\section{Outcome Measurement}

The outcome of interest was dementia and defined as a diagnosis documented with 1 of following ICD-9-CM codes: 290.xx (dementias), 294.1 (dementia in conditions classified elsewhere), 331.0 (Alzheimer disease), and 331.2 (Senile degeneration of brain), with the exception of 290.4 (vascular dementia). We considered the dementia diagnosis to be valid if it was documented at least 3 times in outpatient visits or 1 inpatient hospitalization. The study design is shown in Figure S1.

\section{Other Variables}

The age and sex of subjects were documented at the index date. We also retrieved each subject's medical history within 1 year prior to the index date for an estimation of Charlson Comorbidity Index (CCI) [27] as a proxy of a subject's health status and other comorbidities, including hypertension, insomnia, cerebrovascular disease, dyslipidemia, myocardial infarction, diabetes, depression, psychosis, schizophrenia, delirium, bipolar disorder, and anxiety. In addition, because a previous study indicated that using drugs with anticholinergic effects [28] may be associated with dementia, we also recorded the anticholinergic cognitive burden (ACB) score during the follow-up period as a time-varying (quarterly) covariate. These variables were included in this analysis model.

\section{Statistical Analysis}

Chi-square tests and ANOVAs were used to compare categorical and continuous variables, respectively. Generalized estimating equation (GEE) models (SAS PROC GENMOD) with logit link and first-order autoregressive (AR-1) correlation structure were used to examine the association between distinct half-lives of BZDs and the risk of dementia, taking into account the intraclass correlation between repeated measurements for the same subjects. The GEE models were further adjusted for age, sex, CCI, comorbidity, and time-varying covariates, including the number of quarters since the index date and ACB score. These associations were presented as odds ratios (ORs) with 95\% confidence intervals (CIs). All of the analyses were performed using SAS, version 9.4 (SAS Institute Inc., Cary, NC).

\section{Results}

We identified 417,581 subjects who were 65 years or older and initiated BZDs or z-hypnotics between 2003 and 2012. Approximately $70 \%(n=287,020)$ of these subjects were new users of BZDs or z-hypnotics. We excluded those who had dementia within 1 year before the index date or 1 year after the index date $(n=20,743)$ and those who were diagnosed with vascular dementia or had dementia within 1 year after stoke during follow-up period ( $n=5775)$. Overall, we included data from 260,502 older adults who newly initiated the use of BZDs or z-hypnotics in our analysis (Fig. 1).

As summarized in Table 2, we categorized study subjects into long-acting BZD users ( $n=127,682 ; 49 \%)$, short-acting BZD users $(n=96,657 ; 37 \%)$, and z-hypnotic users $(n=$ $127,682 ; 14 \%)$ according to their first prescription of a BZD or z-hypnotic at the index date. Demographic characteristics at the index date differed significantly across the 3 groups. Overall, short-acting BZD and z-hypnotic users at the index date had higher CCI and were more likely to have other comorbidities than long-acting BZD users. After adjustment for age, sex, CCI, depression, psychosis, delirium, bipolar, anxiety, and ACB scores, GEE models (Table 3) indicated that short-acting BZD and z-hypnotic users had a higher risk of dementia than long-acting BZD users (short-acting BZD users: adjusted odds ratio $(\mathrm{aOR})=1.98,95 \% \mathrm{CI}=1.89$ 


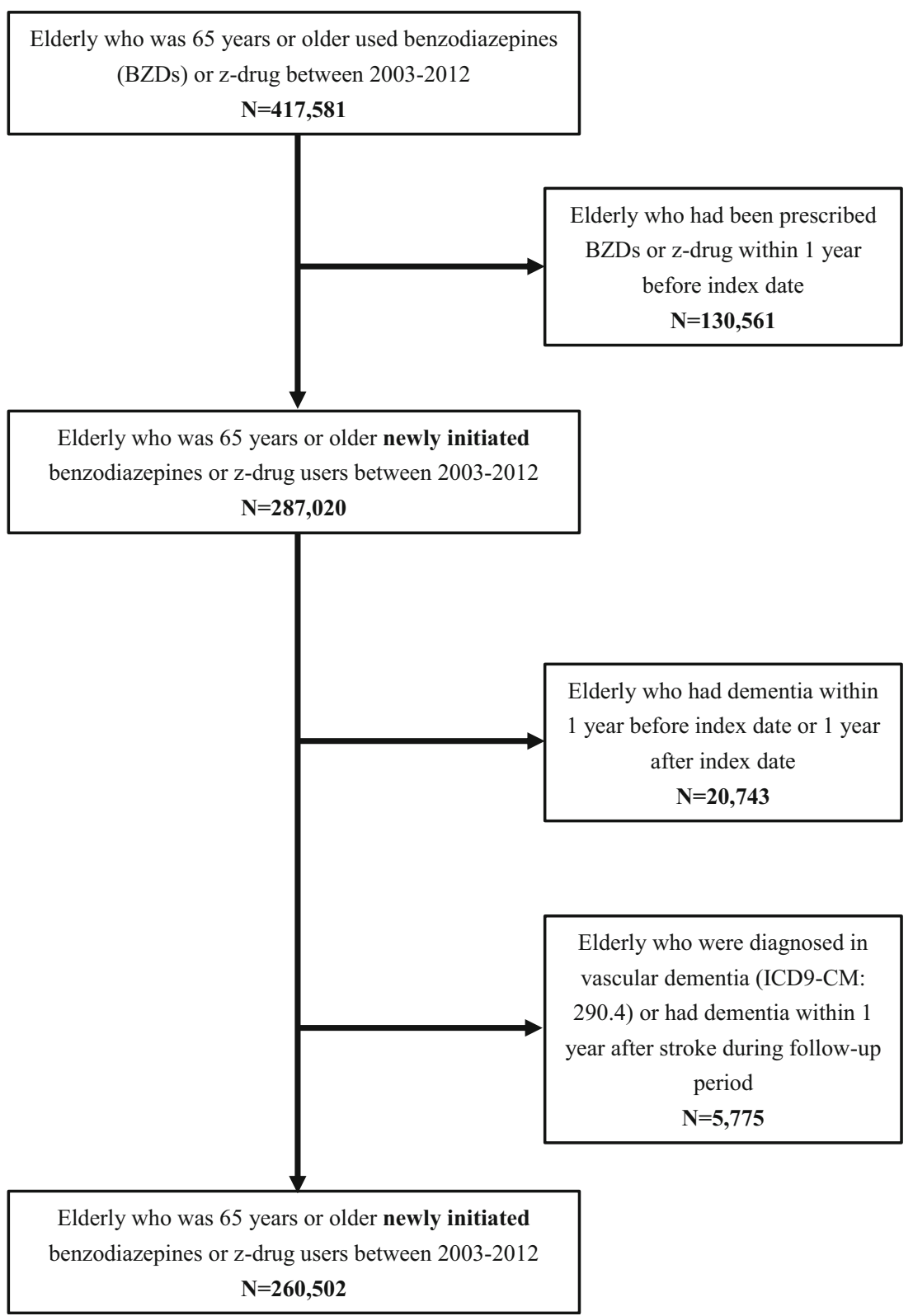

Fig. 1 Study flow chart of inclusion and exclusion of study objects

2.07; z-hypnotic users: $\mathrm{aOR}=1.79,95 \% \mathrm{CI}=1.68-1.91$; long-acting BZD users: $\mathrm{aOR}=1.47,95 \% \mathrm{CI}=1.37-1.58$ ). In addition, long-acting BZDs, short-acting BZDs, and zhypnotics were all associated with a higher risk of dementia than nonusers. GEE models also revealed that the subjects using 2 or more classes of BZDs or z-hypnotics in the same quarter had a higher risk of dementia (long-acting + shortacting BZD users: $\mathrm{aOR}=2.96,95 \% \mathrm{CI}=2.64-3.36$; longacting BZD + z-hypnotic users: $\mathrm{aOR}=2.82,95 \% \mathrm{CI}=2.42-$ 3.28; short-acting BZD + z-hypnotic users: $\mathrm{aOR}=3.25,95 \%$ $\mathrm{CI}=2.97-3.56$ ) than the subjects who only used any 1 class of the drugs; this higher risk was especially true for subjects who concomitantly used long-acting BZDs, short-acting BZDs, and $\mathrm{z}$-hypnotics in the same quarter $(\mathrm{aOR}=4.79,95 \% \mathrm{CI}=$ 3.95-5.81).

In the sensitivity analysis, we changed the definition of drug exposure from using 28 days or more to using 1 day, 7 days, or 14 days or more as cut-points in each quarter. We found that all 3 models yielded results similar to our primary findings (Table 4).

\section{Interpretation}

This population-based retrospective cohort study evaluated the potential associations between long-term and dynamic 
Table 2 Baseline characteristics of study subjects who newly initiated long-acting, short-acting, and z-drug, a subgroup by the kind of prescription in the index date

\begin{tabular}{|c|c|c|c|c|c|c|c|}
\hline & Long-acting $\mathrm{E}$ & $n=127,682)$ & Short-acting $\mathrm{E}$ & $(n=96,657)$ & z-drug $(n=3$ & $36,217)$ & Total $(n=260,502)$ \\
\hline & $n$ & $\%$ & $n$ & $\%$ & $n$ & $\%$ & $p$ value \\
\hline \multicolumn{8}{|l|}{ Age (years) } \\
\hline Mean \pm SD & \multicolumn{2}{|l|}{$72.76 \pm 6.21$} & \multicolumn{2}{|l|}{$73.36 \pm 6.68$} & \multicolumn{2}{|l|}{$73.24 \pm 6.83$} & $<0.001$ \\
\hline $65-75$ & 82,746 & 64.83 & 59,127 & 61.17 & 22,308 & 61.60 & \\
\hline $75-85$ & 38,720 & 30.34 & 30,799 & 31.86 & 11,270 & 31.12 & \\
\hline $85+$ & 6162 & 4.83 & 6731 & 6.96 & 2639 & 7.29 & \\
\hline \multicolumn{8}{|l|}{ Sex } \\
\hline Female & 65,102 & 51.01 & 50,526 & 52.27 & 18,252 & 50.40 & $<0.001$ \\
\hline Male & 62,526 & 48.99 & 46,131 & 47.73 & 17,965 & 49.60 & \\
\hline \multicolumn{8}{|l|}{ CCI } \\
\hline Mean \pm SD & $0.79 \pm 1.19$ & & $1.10 \pm 1.53$ & & $1.22 \pm 1.60$ & & $<0.001$ \\
\hline $0-2$ & 117,256 & 91.87 & 83,839 & 86.74 & 30,618 & 84.54 & \\
\hline $2+$ & 10,372 & 8.13 & 12,818 & 13.26 & 5599 & 15.46 & \\
\hline \multicolumn{8}{|l|}{ Comorbidity } \\
\hline Depression & 887 & 0.69 & 1082 & 1.12 & 438 & 1.21 & $<0.001$ \\
\hline Psychosis & 188 & 0.15 & 391 & 0.40 & 165 & 0.46 & $<0.001$ \\
\hline Schizophrenia & 103 & 0.08 & 255 & 0.26 & 108 & 0.30 & $<0.001$ \\
\hline Delirium & 32 & 0.03 & 47 & 0.05 & 28 & 0.08 & $<0.001$ \\
\hline Bipolar & 81 & 0.06 & 89 & 0.09 & 39 & 0.11 & 0.008 \\
\hline Anxiety & 3488 & 2.73 & 3694 & 3.82 & 1310 & 3.62 & $<0.001$ \\
\hline Hypertension & 51,603 & 40.43 & 44,554 & 46.09 & 17,657 & 48.75 & $<0.001$ \\
\hline Insomnia & 3493 & 2.74 & 4529 & 4.69 & 2554 & 7.05 & $<0.001$ \\
\hline Hyperlipidemia & 14,361 & 11.25 & 12,279 & 12.70 & 5054 & 13.95 & $<0.001$ \\
\hline Myocardial infarction & 842 & 0.66 & 1311 & 1.36 & 445 & 1.23 & $<0.001$ \\
\hline Diabetes & 21,724 & 17.02 & 18,933 & 19.59 & 8094 & 22.35 & $<0.001$ \\
\hline Stroke & 9880 & 7.74 & 9602 & 9.93 & 4071 & 11.24 & $<0.001$ \\
\hline
\end{tabular}

use of BZDs and z-hypnotics and the risk of dementia among individuals aged 65 years and older with a more sophisticated study design than previous studies. The major strength of our study was to capture the long-term and dynamic use of these drugs and thus was able to bridge the knowledge gap involving the switch between different classes of hypnotics that has rarely been done in previous studies. Furthermore, we found that both short-acting and long-acting BZDs led to a greater risk of dementia. To the best of our knowledge, this is the first study considering all these factors to clarify the risk of BZDs and z-hypnotics on dementia. Compared to the nonusers, older people using BZDs (either long-acting or short-acting ones) or z-hypnotics for more than 28 days during every quarter of the year had a greater risk of dementia ( $47 \%$ greater for long-acting BZDs, $98 \%$ for short-acting BZDs, and $79 \%$ for zhypnotics). We also found that the elderly population that used more than 1 class of sedatives (either BZDs or z-hypnotics) had a higher risk of dementia than the population that used a single drug class. The risk of dementia was 4.79-fold higher in long-acting BZD + short-acting BZD + z-hypnotic (3 combined) users than nonusers. Those with the combined use of short-acting BZDs and z-hypnotics had a 3.25-fold higher risk of dementia than the nonusers.

Our study showed that short-acting BZDs and z-hypnotics users had a higher risk of dementia than long-acting BZDs users. Previous studies have shown that long-acting BZDs (half-life $>20 \mathrm{~h}$ ) are at higher risk than short-acting ones in the risk of dementia development [8-10]. Takada et al. [8] conducted a study and established the associations between BZD use and dementia from 3 large databases and reported that long-acting BZD use was strongly associated with an increased risk of dementia than short-acting BZD use. A case-control study in Quebec enrolled 1796 people with incident Alzheimer's disease matched with 7184 controls and found that ever use of BZDs was associated with a $51 \%$ higher risk of Alzheimer's disease [9]. The association between Alzheimer's disease and BZD use was stronger for longacting BZDs $(\mathrm{HR}=1.70,95 \% \mathrm{CI}=1.46$ to 1.98$)$ than shortacting ones $(\mathrm{HR}=1.43,95 \% \mathrm{CI}=1.27$ to 1.61$)$. The ThreeCity Study from France also reported a substantial risk of 
Table 3 Association between distinct half-life of BZD and z-drug and the risk of dementia adjusted for quarter, age, sex, CCI, ACB, and comorbidities

\begin{tabular}{|c|c|c|c|c|}
\hline & \multicolumn{2}{|c|}{ Unadjusted } & \multicolumn{2}{|c|}{ Adjusted } \\
\hline & OR & $95 \% \mathrm{CI}$ & OR & $95 \% \mathrm{CI}$ \\
\hline \multicolumn{5}{|l|}{ BZD } \\
\hline Nonuser & ref & & ref & \\
\hline Long-acting BZD (L) & 1.48 & $1.37-1.59$ & 1.47 & $1.37-1.58$ \\
\hline Short-acting BZD (S) & 2.31 & $2.21-2.41$ & 1.98 & $1.89-2.07$ \\
\hline z-drug $(Z)$ & 1.92 & $1.81-2.05$ & 1.79 & $1.68-1.91$ \\
\hline $\mathrm{L}+\mathrm{S}$ & 3.18 & $2.83-3.58$ & 2.96 & $2.64-3.36$ \\
\hline$L+Z$ & 2.88 & $2.48-3.35$ & 2.82 & $2.42-3.28$ \\
\hline$S+Z$ & 3.77 & $3.46-4.10$ & 3.25 & $2.97-3.56$ \\
\hline $\mathrm{L}+\mathrm{S}+\mathrm{Z}$ & 5.04 & $4.18-6.09$ & 4.79 & $3.95-5.81$ \\
\hline \multicolumn{5}{|l|}{ Others } \\
\hline Quarter & & & 1.05 & $1.05-1.05$ \\
\hline \multicolumn{5}{|l|}{ Age (years) } \\
\hline $65-75$ & & & ref & \\
\hline $75-85$ & & & 2.59 & $2.51-2.68$ \\
\hline $85+$ & & & 5.32 & $5.02-5.63$ \\
\hline \multicolumn{5}{|l|}{ Sex } \\
\hline Female & & & ref & \\
\hline Male & & & 0.92 & $0.89-0.95$ \\
\hline \multicolumn{5}{|l|}{ CCI } \\
\hline $0-2$ & & & ref & \\
\hline $2+$ & & & 1.44 & $1.42-1.46$ \\
\hline ACB score & & & 1.34 & $1.27-1.41$ \\
\hline \multicolumn{5}{|l|}{ Comorbidity } \\
\hline Depression & & & 1.05 & $0.90-1.22$ \\
\hline Psychosis & & & 1.74 & $1.39-2.18$ \\
\hline Delirium & & & 3.37 & $2.00-5.68$ \\
\hline Bipolar & & & 1.97 & $1.37-2.82$ \\
\hline Anxiety & & & 0.89 & $0.81-0.97$ \\
\hline
\end{tabular}

$\mathrm{CCI}=$ Charlson Comorbidity Index; $\mathrm{ACB}=$ anticholinergic cognitive burden

long-acting BZDs in dementia development (HR $=1.62,95 \%$ $\mathrm{CI}=1.11$ to 2.37 ) through a prospective study design, and the risk of short-acting BZDs was only marginal ( $\mathrm{HR}=1.05,95 \%$ $\mathrm{CI}=0.85$ to 1.3 ) [10]. One potential explanation for the abovementioned discrepancies could be that short-acting BZD users and z-hypnotic users in our study were older and had more multimorbidity than the long-acting BZD users. Moreover, long-acting BZDs have been considered potentially inappropriate medications for older adults $[29,30]$, and therefore, the utilization may have been reduced in older populations. This phenomenon could imply that physicians may prefer short-acting BZDs or z-hypnotics for older people because of their older age and multiple comorbid conditions, as well as the strong risk of falls associated with long-acting BZDs.
A number of studies have shown the adverse cognitive and memory effects of BZDs and z-hypnotics [31-34], including both nonamnestic and amnestic cognitive impairment. Both BZDs and z-hypnotics are gamma-aminobutyric acid (GABA)-ergic agents and function as positive allosteric modulators of GABA-A receptors [6, 35-39]. After GABA-A receptors are bound by BZDs or z-hypnotics, the chloride ion channel is opened, resulting in central nervous system (CNS) inhibition and subsequent adverse effects. Previous studies indicated that BZD use might decrease the expression of GABA-A receptors in synapses, which could decrease postsynaptic inhibition [40, 41]. In addition, BZD use would also affect GABA-A receptor-mediated synaptic currents in the hippocampal cornu ammonis area 1 (CA1) pyramidal neurons, which may affect the balance of excitation and inhibition in the CNS [41, 42]. In addition to the synaptic effects of BZDs and z-hypnotics, both BZDs and z-hypnotics may bind to BZD-1 receptors, 1 of the subtypes of BZD receptors in the CNS that has been associated with sedative effects and anterograde amnesia [36].

Although many studies have shown associations between BZDs and z-hypnotics and dementia, the exact mechanisms remain unclear. A previous study suggested that the BZDinduced inhibitory GABA effect in the CNS may reduce the activity of beta secretase and gamma secretase, which are protective against cognitive declines [43]. However, new therapeutic agents inhibiting gamma secretase have not shown significant effects on dementia compared to placebo [44, 45]. Moreover, some unfavorable effects of the gamma secretase inhibitor semagacestat (LY-450139) have been reported. Another hypothesis is that "excitotoxicity" may occur in the overactivated CNS [46]. The potential calm-down effects of BZDs in the CNS may play some role in dementia protection, but it is difficult to evaluate the excitation toxicity of each individual drug. Although the protective effects of BZDs against cognitive impairment had been proposed based on findings in the laboratory, the real-world data was showing the opposite effects in that BZD use was associated with a higher risk of dementia. Further study evaluating the pharmacological effects of BZDs and z-hypnotics on cognitive function is needed to confirm the roles of these pharmacological agents in cognitive health.

The strength of this study included the large study sample size and the dynamic relationship between BZD and zhypnotic use, as well as the special consideration of the halflives of individual drugs and their concomitant use. The study design enabled us to explore the complex interrelationship between real-world patterns of BZD and z-hypnotic use and the risk of dementia. However, some limitations still exist despite all the effort that went into the study. First, some factors related to cognitive function, such as education, smoking, alcohol consumption, family history, Apo-E4, and baseline cognitive function, were not available in the dataset. 
Table 4 Association between distinct half-life of BZD and z-drug and the risk of dementia with 1 day, 7 days, and 14 days cut-point of drug exposure

\begin{tabular}{|c|c|c|c|c|c|c|}
\hline \multirow[t]{2}{*}{ Cut-point } & \multicolumn{2}{|l|}{1 day } & \multicolumn{2}{|c|}{7 days } & \multicolumn{2}{|c|}{14 days } \\
\hline & OR & $95 \% \mathrm{CI}$ & OR & $95 \% \mathrm{CI}$ & OR & $95 \% \mathrm{CI}$ \\
\hline \multicolumn{7}{|l|}{ BZD } \\
\hline Nonuser & ref & & ref & & ref & \\
\hline Long-term BZD (L) & 1.17 & $1.10-1.24$ & 1.42 & $1.33-1.52$ & 1.51 & $1.41-1.62$ \\
\hline Short-term BZD (S) & 2.03 & $1.94-2.12$ & 2.15 & $2.06-2.25$ & 2.15 & $2.05-2.24$ \\
\hline Z-drug (Z) & 1.9 & $1.78-2.02$ & 1.92 & $1.80-2.04$ & 1.93 & $1.81-2.05$ \\
\hline $\mathrm{L}+\mathrm{S}$ & 2.96 & $2.74-3.20$ & 3.64 & $3.34-3.97$ & 3.51 & $3.19-3.88$ \\
\hline$L+Z$ & 2.62 & $2.33-2.95$ & 3.01 & $2.65-3.42$ & 2.94 & $2.56-3.38$ \\
\hline$S+Z$ & 4.05 & $3.79-4.34$ & 4.23 & $3.94-4.53$ & 3.94 & $3.65-4.25$ \\
\hline $\mathrm{L}+\mathrm{S}+\mathrm{Z}$ & 6.07 & $5.52-6.67$ & 6.67 & $5.95-7.46$ & 6.22 & $5.43-7.13$ \\
\hline \multicolumn{7}{|l|}{ Others } \\
\hline Quarter & 1.05 & $1.05-1.05$ & 1.05 & $1.05-1.05$ & 1.05 & $1.05-1.05$ \\
\hline \multicolumn{7}{|l|}{ Age (years) } \\
\hline $65-75$ & ref & & ref & & ref & \\
\hline $75-85$ & 2.64 & $2.55-2.73$ & 2.63 & $2.54-2.72$ & 2.61 & $2.53-2.70$ \\
\hline $85+$ & 5.45 & $5.15-5.77$ & 5.43 & $5.13-5.74$ & 5.38 & $5.09-5.70$ \\
\hline \multicolumn{7}{|l|}{ Sex } \\
\hline Female & ref & & ref & & ref & \\
\hline Male & 0.93 & $0.90-0.96$ & 0.93 & $0.90-0.96$ & 0.92 & $0.89-0.95$ \\
\hline \multicolumn{7}{|l|}{ CCI } \\
\hline $0-2$ & ref & & ref & & ref & \\
\hline $2+$ & 1.34 & $1.27-1.42$ & 1.33 & $1.26-1.40$ & 1.33 & $1.26-1.40$ \\
\hline ACB score & 1.40 & $1.38-1.42$ & 1.40 & $1.38-1.43$ & 1.42 & $1.40-1.44$ \\
\hline \multicolumn{7}{|l|}{ Comorbidity } \\
\hline Depression & 1.04 & $0.90-1.21$ & 1.00 & $0.86-1.16$ & 1.01 & $0.87-1.17$ \\
\hline Psychosis & 1.81 & $1.45-2.28$ & 1.74 & $1.39-2.19$ & 1.72 & $1.37-2.16$ \\
\hline Delirium & 3.30 & $1.94-5.64$ & 3.25 & $1.91-5.53$ & 3.28 & $1.94-5.55$ \\
\hline Bipolar & 1.97 & $1.37-2.83$ & 1.91 & $1.33-2.75$ & 1.92 & $1.34-2.76$ \\
\hline Anxiety & 0.87 & $0.79-0.94$ & 0.85 & $0.78-0.93$ & 0.86 & $0.78-0.93$ \\
\hline
\end{tabular}

$\mathrm{CCI}=$ Charlson Comorbidity Index $\mathrm{ACB}=$ anticholinergic cognitive burden

However, this is the common situation with claims data that is universal across other studies. Second, we excluded vascular dementia in this study due to the small reported case number and the lack of brain images to confirm the diagnosis. However, other types of dementia with specific etiologies may have been included in the diagnostic entity of dementia because most physicians may not clearly differentiate those patients in their daily practice. However, the case number of other types of dementia was small, so the results of the study may remain similar with or without specific classification of the etiology. Third, similar to all claims data-based studies, adherence to the medications for the study subjects was unknown, which may result in overestimating the adverse effects of BZDs and z-hypnotics. Fourth, a 10-year (2003-2012) panel of claims for the analysis may not be long enough to confirm the risk of dementia following the use of these pharmaceutical agents. Fifth, the algorithm we adopted to identify dementia via discharge diagnosis at inpatient visits may not have guaranteed that these cases had subsequent dementia diagnoses at outpatient visits. We thus conducted sensitivity analyses by excluding those discharged with a dementia diagnosis but without a dementia diagnosis in subsequent outpatient visits $(n=1326)$ (Table S1). The results were consistent between the original model (dementia cases, $n=$ 23,919 ) and sensitivity analyses (dementia cases, $n=$ 22,593). Therefore, we believe our findings are robust.

\section{Conclusions}

In conclusion, the results of this study suggested that the use of BZDs and/or z-hypnotics may increase the risk of dementia 
in a 10-year follow-up period, and the concomitant use of these agents significantly increases the risk. Moreover, shortacting BZDs and z-hypnotics were of greater risk of dementia than long-acting BZDs. Further intervention studies are needed to confirm the causal relationships between the use of BZDs and z-hypnotics and the risk of dementia.

Acknowledgments The authors thank the National Health Insurance Association and National Health Research Institutes for making available the databases for this study. The content of this article, however, in no way represents any official position of the National Health Insurance Association or National Health Research Institutes. The authors had full access to all the data in the study and took responsibility for the integrity of the data and the accuracy of the data analysis.

Author Contributions All the authors drafted the article, revised it critically for important intellectual content, and approved the final version to be published. LY Tseng, ST Huang, and FY Hsiao designed the research and wrote the paper. LY Tseng performed the literature search. ST Huang performed the data analysis. FY Hsiao provided critical methodological and statistical inputs. LY Tseng, LN Peng, and LK Chen contributed to the clinical interpretation. FY Hsiao is the guarantor.

Funding Information Dr. Hsiao FY received research assistantships from the research projects (MOST 104-2410-H-002-225-MY3) sponsored by the Ministry of Science and Technology Taiwan and the research projects (MOST 107-2634-F-010-001 and 108-2634-F-010-001) sponsored by the Ministry of Science and Technology Taiwan. Dr. Chen LK received research assistantships from the research projects (MOST 107-2634-F010-001 and 108-2634-F-010-001) sponsored by the Ministry of Science and Technology Taiwan.

\section{References}

1. Taylor DJ, Mallory LJ, Lichstein KL, Durrence HH, Riedel BW, Bush AJ. Comorbidity of chronic insomnia with medical problems. Sleep, 30(2), 213-218 (2007).

2. Bachhuber MA, Hennessy S, Cunningham CO, Starrels JL. Increasing benzodiazepine prescriptions and overdose mortality in the United States, 1996-2013. American Journal of Public Health, 106(4), 686-688 (2016).

3. Olfson M, King M, Schoenbaum M. Benzodiazepine use in the United States. JAMA Psychiatry, 72(2), 136-142 (2015).

4. Hsiao F-Y, Hsieh P-H, Gau C-S. Ten-year trend in prescriptions of z-hypnotics among the elderly: a nationwide, cross-sectional study in Taiwan. Journal of Clinical Gerontology and Geriatrics, 4(2), 37-41 (2013).

5. Brandt J, Leong C. Benzodiazepines and Z-Drugs: An Updated Review of Major Adverse Outcomes Reported on in Epidemiologic Research. Drugs in R\&D, 17(4), 493-507 (2017).

6. Smink BE, Egberts AC, Lusthof KJ, Uges DR, De Gier JJ. The relationship between benzodiazepine use and traffic accidents. $C N S$ Drugs, 24(8), 639-653 (2010).

7. Donnelly K, Bracchi R, Hewitt J, Routledge PA, Carter B. Benzodiazepines, Z-drugs and the risk of hip fracture: A systematic review and meta-analysis. PLoS One, 12(4), e0174730 (2017).

8. Takada M, Fujimoto M, Hosomi K. Association between benzodiazepine use and dementia: data mining of different medical databases. International Journal of Medical Sciences, 13(11), 825 (2016).
9. de Gage SB, Moride Y, Ducruet T et al. Benzodiazepine use and risk of Alzheimer's disease: case-control study. BMJ, 349, g5205 (2014).

10. Shash D, Kurth T, Bertrand M et al. Benzodiazepine, psychotropic medication, and dementia: A population-based cohort study. Alzheimer's \& Dementia: the journal of the Alzheimer's Association, 12(5), 604-613 (2016).

11. Hsiao F-Y, Peng L-N, Lin M-H, Chen L-K. Dose-responsive effect of psychotropic drug use and subsequent dementia: a nationwide propensity score matched case-control study in Taiwan. Journal of the American Medical Directors Association, 15(7), 509-513 (2014).

12. Shih H-I, Lin C-C, Tu Y-F et al. An increased risk of reversible dementia may occur after zolpidem derivative use in the elderly population: a population-based case-control study. Medicine, 94(17) (2015).

13. Zhong G, Wang Y, Zhang Y, Zhao Y. Association between benzodiazepine use and dementia: a meta-analysis. PLoS One, 10(5), e0127836 (2015).

14. Islam MM, Iqbal $U$, Walther $B$ et al. Benzodiazepine use and risk of dementia in the elderly population: a systematic review and metaanalysis. Neuroepidemiology, 47(3-4), 181-191 (2016).

15. Federico A, Tamburin S, Maier A et al. Multifocal cognitive dysfunction in high-dose benzodiazepine users: a cross-sectional study. Neurological Sciences: official journal of the Italian Neurological Society and of the Italian Society of Clinical Neurophysiology, 38(1), 137-142 (2017).

16. Zhang Y, Zhou XH, Meranus DH, Wang L, Kukull WA. Benzodiazepine Use and Cognitive Decline in Elderly With Normal Cognition. Alzheimer Disease and Associated Disorders, 30(2), 113-117 (2016).

17. Chung JK, Nakajima S, Shinagawa $S$ et al. Benzodiazepine Use Attenuates Cortical beta-Amyloid and is Not Associated with Progressive Cognitive Decline in Nondemented Elderly Adults: A Pilot Study Using F(18)-Florbetapir Positron Emission Tomography. The American Journal of Geriatric Psychiatry: official journal of the American Association for Geriatric Psychiatry, 24(11), 1028-1039 (2016).

18. Puustinen J, Nurminen J, Vahlberg T et al. CNS medications as predictors of precipitous cognitive decline in the cognitively disabled aged: a longitudinal population-based study. Dementia and Geriatric Cognitive Disorders Extra, 2(1), 57-68 (2012).

19. Puustinen J, Nurminen J, Lopponen M et al. Use of CNS medications and cognitive decline in the aged: a longitudinal populationbased study. BMC Geriatrics, 11, 70 (2011).

20. Bourgeois J, Elseviers MM, Van Bortel L, Petrovic M, Vander Stichele RH. The impact of chronic benzodiazepine use on cognitive evolution in nursing home residents. Human Psychopharmacology, 30(2), 85-93 (2015).

21. Lai YR, Yang YS, Tsai ML, Huang CN, Chiou JY. Benzodiazepine $\&$ nonbenzodiazepine prescriptions for Taiwanese elderly with type 2 diabetes contributes to cognitive dysfunction. International Psychogeriatrics, 26(10), 1719-1727 (2014).

22. Wu CS, Wang SC, Chang IS, Lin KM. The association between dementia and long-term use of benzodiazepine in the elderly: nested case-control study using claims data. The American Journal of Geriatric Psychiatry: official journal of the American Association for Geriatric Psychiatry, 17(7), 614-620 (2009).

23. Wu CS, Ting TT, Wang SC, Chang IS, Lin KM. Effect of benzodiazepine discontinuation on dementia risk. The American Journal of Geriatric Psychiatry: official journal of the American Association for Geriatric Psychiatry, 19(2), 151-159 (2011).

24. Hsiao F-Y, Yang C-L, Huang Y-T, Huang W-F. Using Taiwan's national health insurance research databases for pharmacoepidemiology research. Journal of Food and Drug Analysis, 15(2) (2007). 
25. Imfeld P, Bodmer M, Jick SS, Meier CR. Proton Pump Inhibitor Use and Risk of Developing Alzheimer's Disease or Vascular Dementia: A Case-Control Analysis. Drug Safety, 41(12), 13871396 (2018).

26. Ferner R. Handbook of drug interactions: a clinical and forensic guide. (Eds) (Wiley Online Library, 2012)

27. Charlson ME, Pompei P, Ales KL, MacKenzie CR. A new method of classifying prognostic comorbidity in longitudinal studies: development and validation. Journal of Chronic Diseases, 40(5), 373383 (1987).

28. Hsu W-H, Wen Y-W, Chen L-K, Hsiao F-Y. Comparative Associations Between Measures of Anti-cholinergic Burden and Adverse Clinical Outcomes. The Annals of Family Medicine, 15(6), 561-569 (2017).

29. Laroche M-L, Charmes J-P, Merle L. Potentially inappropriate medications in the elderly: a French consensus panel list. European Journal of Clinical Pharmacology, 63(8), 725-731 (2007).

30. O'Mahony D, Gallagher P, Ryan C et al. STOPP \& START criteria: a new approach to detecting potentially inappropriate prescribing in old age. European Geriatric Medicine, 1(1), 45-51 (2010).

31. Roth T, Roehrs T, Wittig R, Zorick F. Benzodiazepines and memory. British Journal of Clinical Pharmacology, 18 Suppl 1, 45 s-49 s (1984).

32. Lister RG. The amnesic action of benzodiazepines in man. Neuroscience \& Biobehavioral Reviews, 9(1), 87-94 (1985).

33. Curran HV. Tranquillising memories: a review of the effects of benzodiazepines on human memory. Biological Psychology, 23(2), 179-213 (1986).

34. Tannenbaum C, Paquette A, Hilmer S, Holroyd-Leduc J, Carnahan R. A systematic review of amnestic and non-amnestic mild cognitive impairment induced by anticholinergic, antihistamine, GABAergic and opioid drugs. Drugs \& Aging, 29(8), 639-658 (2012).

35. Pariente A, de Gage SB, Moore N, Bégaud B. The benzodiazepinedementia disorders link: current state of knowledge. CNS Drugs, 30(1), 1-7 (2016).

36. Griffin III CE, Kaye AM, Bueno FR, Kaye AD. Benzodiazepine pharmacology and central nervous system-mediated effects. The Ochsner Journal, 13(2), 214-223 (2013).
37. Gunja N. In the Zzz zone: the effects of Z-drugs on human performance and driving. Journal of Medical Toxicology, 9(2), 163-171 (2013).

38. Gunja N. The clinical and forensic toxicology of Z-drugs. Journal of Medical Toxicology, 9(2), 155-162 (2013).

39. Paul L Soto, Nancy A Ator, Sundari K Rallapalli, Poonam Biawat, Terry Clayton, James M Cook et al. Allosteric Modulation of GABAA Receptor Subtypes: Effects on Visual Recognition and Visuospatial Working Memory in Rhesus Monkeys Neuropsychopharmacology. 38(11): 2315-2325.(2013)

40. Tija C. Jacob, Guido Michels, Liliya Silayeva, Julia Haydon, Francesca Succol, and Stephen J. Moss. Benzodiazepine treatment induces subtype-specific changes in GABAA receptor trafficking and decreases synaptic inhibition. PNAS 109 (45) 1859518600.(2012)

41. Lilly SM, Zeng XJ, Tietz EI. Role of protein kinase A in GABAA receptor dysfunction in $\mathrm{CA} 1$ pyramidal cells following chronic benzodiazepine treatment. J Neurochem. 85(4):988-98.(2003)

42. Kun Xiang and Elizabeth I. Tietz. Chronic Benzodiazepine-induced reduction in GABAA receptor-mediated synaptic currents in hippocampal CA1 pyramidal neurons prevented by prior nimodipine injection. Neuroscience. 157(1): 153-163.(2008)

43. Fastbom J, Forsell Y, Winblad B. Benzodiazepines may have protective effects against Alzheimer disease. Alzheimer Disease and Associated Disorders, 12(1), 14-17 (1998).

44. Churcher I, Ashton K, Butcher JW et al. A new series of potent benzodiazepine $\gamma$-secretase inhibitors. Bioorganic \& Medicinal Chemistry Letters, 13(2), 179-183 (2003).

45. Doody RS, Raman R, Farlow M et al. A phase 3 trial of semagacestat for treatment of Alzheimer's disease. New England Journal of Medicine, 369(4), 341-350 (2013).

46. Dong XX, Wang Y, Qin ZH. Molecular mechanisms of excitotoxicity and their relevance to pathogenesis of neurodegenerative diseases. Acta Pharmacologica Sinica, 30(4), 379-387 (2009).

Publisher's Note Springer Nature remains neutral with regard to jurisdictional claims in published maps and institutional affiliations. 